\begin{abstract}
The goal of this work is to quantify the Van der Waals interactions in systems involving gas hydrates. Gas hydrates are crystalline compounds that are often encountered in oil and gas industry, where they pose problems (pipeline plugging, ...) and represent opportunities (energy resources, gas transport, ...). We focus on methane hydrate, which is the most common one, and calculate its Hamaker constant. Two methods are used and lead to results in good agreement. The Hamaker, microscopic, approach gives a first estimate of the Hamaker constant of $4.59 \times 10^{-21} \mathrm{~J}$ for the hydrate-water-hydrate system. The Lifshitz, macroscopic, method used in combination with the Kramers-Kronig relationship gives a value of $8.25 \times 10^{-21} \mathrm{~J}$. The Hamaker constant is also computed for three phases systems (gas hydrate clathrate and liquid water with ice, dodecane, quartz, sapphire, teflon, metals). The interaction potential in different geometrical configurations is then calculated by a hybrid method and various cases of practical interest are studied.
\end{abstract}

Author Keywords: Methane gas hydrate; Hamaker constant; Agglomeration; Dielectric response function; Van der Waals interaction potential. 


\title{
Van der Waals interactions in systems involving
}

\author{
gas hydrates
}

[O. Bonnefoy*, F. Gruy, J.-M. Herri]

Centre SPIN, UMR CNRS 5148,

Ecole des Mines de Saint-Etienne,

158 cours Fauriel, F-42023 Saint-Etienne, France

* olivier.bonnefoy@emse.fr

June 8, 2004

This article is organized as follows : in the introduction, we briefly present the hydrate crystalline structure and the role of hydrates in oil-and gas industry. The introduction reminds also some basic concepts concerning Van der Waals forces. Then, in the following 4 sections, we successively apply the Hamaker and Lifshitz theories (theory and calculation) to propose a value for the Hamaker constant. Finally, we hybrid these two methods and compute the Van der Waals interaction potential for two spherical hydrate particles. Some comments and a conclusion end the article.

\section{Introduction}

Context Gas hydrate clathrates are crystalline compounds composed of host gas molecules trapped in a lattice of water molecules. In industrial contexts, the host gas is commonly methane, ethane or carbon dioxide but clathrates may form with other gases like light alcanes (up to $\mathrm{C}_{4}$ ), hydrogen sulfide, dioxygen, dinitrogen and some rare gases (argon, neon, krypton, ... ). Literature is abundant on gas hydrates and the novice can safely refer to Sloan's book [1] for a detailed presentation of these crystals. Among the industrial contexts where they appear, we shall cite : hydrate plugs obstructing oil- or gas-pipelines, energy resources in form of methane hydrates trapped in permafrost or submarine sediments, natural gas transport in form of a slurry or gas separation by fractionated crystallization. The intelligent exploitation of hydrates in these contexts require a considerable amount of scientific data and the research efforts of many nations (United States, Russia, France, Germany, Great-Britain, Japan and China, to cite the most active ones) greatly contribute to this goal. From 
this perspective, it seems to us that the knowledge of the Hamaker constant of systems involving gas hydrates is a key point, in particular for those who wish to predict the agglomeration behaviour of these systems. Since we do not expect the Hamaker constant to depend too much from the host gas nature, we restrict our study to methane hydrates.

Interactions between molecules Before going into detail, let us remember some basic concepts about the interactions between molecules. When electrostatic interactions are absent or negligible, molecular interactions are of two natures. Firstly, we find the short range, repulsive interactions due to the electronic clouds that cannot overlap. Different models describe this interaction. Among them, let us quote the hard sphere potential, exponential decay potential and power law potential. Secondly, we have the long range, attractive interactions. Three of them are known and they all have a potential that varies as the inverse sixth power of the intermolecular distance.

- The orientation interaction, or Keesom interaction, tends to correlate the relative orientation of two polar molecules. The Keesom potential [2] depends on the molecule dipole moments $\mu_{i}$ :

$$
u_{(r)}=\frac{-1}{\left(4 \pi \epsilon_{0}\right)^{2}} \cdot \frac{\mu_{1}^{2} \mu_{2}^{2}}{3 k T . r^{6}}
$$

where $\epsilon_{0}=8.854 \times 10^{-12} C^{2} . J^{-1} \cdot m^{-1}$ is the vacuum permittivity and $k=1.38054 \times 10^{-23}$ J. $K^{-1}$ is the Boltzmann constant.

- The induction interaction, or Debye interaction, is observed between an apolar molecule and a polar molecule. The latter creates an electric field that induces a dipole moment on the apolar but polarisable molecule. The Debye potential [3] is

$$
u_{(r)}=\frac{-1}{\left(4 \pi \epsilon_{0}\right)^{2}} \cdot \frac{\alpha_{1} \mu_{2}^{2}+\alpha_{2} \mu_{1}^{2}}{r^{6}}
$$

- The dispersion interaction, or London interaction is observed between two apolar molecules and was first explained by Eisenschitz \& London [4]. The time fluctuations of the electronic cloud density, produce a transient dipole moment, which average is zero. At every moment, however, this dipole moment generates an electric field that acts on the second molecule and induces a dipole moment. For two molecules of polarisability $\alpha_{i}$ and first ionisation frequency $\nu_{i}$, the London potential [5] is

$$
u_{(r)}=\frac{-1}{\left(4 \pi \epsilon_{0}\right)^{2}} \cdot \frac{3 \alpha_{1} \alpha_{2} \cdot h \nu_{1} \nu_{2}}{2\left(\nu_{1}+\nu_{2}\right) \cdot r^{6}}
$$

where $h=6.626 \times 10^{-34} \mathrm{~J} . \mathrm{s}$ is the Planck constant.

Retardation effect We should note the existence of a retardation effect of the dispersion interaction. When two apolar molecules are separated by a relatively long distance, the time required for the electric field to travel $(t=2 * L / c)$ can become comparable to the fluctuation period of the dipole itself ${ }^{1}$. In this

\footnotetext{
${ }^{1}$ The revolution time of the electron of the Bohr atom is equal to the inverse of the first ionisation frequency which is about $3 \times 10^{15} \mathrm{~s}^{-1}$.
} 
case, the field that comes back on the first molecule finds that the instantaneous dipole direction changed and produces a smaller attraction. Casimir \& Polder [6] showed that the retardation effect is negligible for distances below $\approx 10 \mathrm{~nm}$ and that, for distances above $\approx 100 \mathrm{~nm}$, this effect creates a dependence in $-1 / r^{7}$ instead of $-1 / r^{6}$.

Van der Waals interaction potential The Van der Waals interaction(s) is the generic name for the three attractive interactions. Its potential is the sum of the corresponding potentials

$$
u_{(r)}=-C / r^{6}
$$

where $C$ is the Van der Waals constant, positive and equal to the sum of the three contributions

$$
C=\frac{1}{\left(4 \pi \epsilon_{0}\right)^{2}} \cdot\left[\frac{\mu_{1}^{2} \mu_{2}^{2}}{3 k T}+\alpha_{1} \mu_{2}^{2}+\alpha_{2} \mu_{1}^{2}+\frac{3 \alpha_{1} \alpha_{2} \cdot h \nu_{1} \nu_{2}}{2\left(\nu_{1}+\nu_{2}\right)}\right]
$$

The table 1 gives these constants for different pairs of molecules. Data are from Israelachvili [7].

\section{Hamaker approach : theory}

This Hamaker approach to calculate the interactions between macroscopic bodies is also known as the microscopic approach since it focuses at the molecular structure scale.

\subsection{Hypothesis}

In 1937, Hamaker [8] proposed a method to calculate the interaction force between two macroscopic bodies (1 and 2). He made two assumptions

- The retardation effect of the dispersion interaction is neglected, whatever the distances are,

- The interaction potential between two molecules keeps the same form even if other molecules surround them. This is often called additivity assumption.

Hamaker used a potential in $1 / r^{6}$ to describe the Van der Waals interaction forces between two molecules and the expression (2) to calculate the Van der Waals constant $C$. Then he summed up the pair-potentials between molecules in body (1) and molecules in body (2).

\subsection{Interaction potential}

The Hamaker method leads to expressions in form of a product of two functions $A$ and $f$. The function $f$ depends only on the shape of the bodies and on the separation distance $d \geq 0$. The table 9 gives this function for different 
geometries and for different separation distances (the plate width is noted $e$ ). The function $A$ is the Hamaker constant. Since we use the Hamaker method, we write $H$ as exponent. We can estimate the interaction potential $U$ by the expression

$$
U_{(d)}^{H}=-A^{H} \cdot f_{(d, g e o m e t r y)}
$$

Tadmor [10] proposes a expression for $f$ in the case of two spherical shells, one spherical shell and a sphere as well as one spherical shell and a half space. These expressions may be of interest for example in the case of water droplets undergoing crystallisation into hydrate.

For a sphere in a cylindrical pore, the work of Bhattacharjee \& Sharma [11] reviews the existing calculation methods. Zeman \& Wales [12] as well as Papadopoulos \& Kuo [13] proposed numerical methods to solve this question. However, they require prohibitive computing time. Sharma uses the two Hamaker assumptions to simplify these expressions. He studies the limiting case of a particle near the revolution axis and near the pore wall. This study is of major interest to evaluate the interaction potential between a particle and the wall of a porous medium. In the particular case of an infinite cylindrical pore containing a spherical particle near the wall, we have the following approximation

$$
f_{(d, \text { geometry })}=\frac{\lambda^{3}}{3\left[(1-\eta(1-\lambda))^{2}-\lambda^{2}\right] \cdot \sqrt{(1-\eta(1-\lambda))^{2} \cdot(1+\eta(1-\lambda))-\lambda^{2}}}
$$

where $\lambda=\frac{R_{\text {pore }}}{R_{\text {particle }}}$ and $\eta=\frac{r}{R_{\text {pore }}-R_{\text {particle }}}$ with $R_{\text {pore }}, R_{\text {particle }}$ and $r$ representing respectively the pore radius, the particle radius and the distance between the particle center and the revolution axis of the pore.

\subsection{Hamaker constant according to Hamaker}

When bodies (1) and (2) are separated by vacuum, the Hamaker constant $A^{H}$ is

$$
A_{12}^{H}=\pi^{2} \cdot C_{12} \cdot \rho_{1} \cdot \rho_{2}
$$

where $C_{12}$ is the Van der Waals constant for the pair of molecules $(1,2)$ and $\rho_{i}$ the molecular density ${ }^{2}$ constituting the body (i). When bodies (1) and (2) are separated by a medium (3), we use again the additivity assumption and the Hamaker constant $A^{H}$ becomes

$$
A_{132}^{H}=A_{12}^{H}+A_{33}^{H}-A_{13}^{H}-A_{23}^{H}
$$

The Hamaker constant depends only on the intrinsic properties of the materials. It usually ranges between $10^{-21}$ and $10^{-19} \mathrm{~J}$. The attractive or repulsive force is simply the derivative of the interaction potential with respect to the separation distance between the two bodies.

\footnotetext{
${ }^{2}$ The molecular density, expressed in molecules $/ \mathrm{m}^{3}$, is equal to $\rho=\frac{N_{A} \cdot \rho_{V}}{\dot{M}}$ where $\rho_{V}$ is the density $\left(\mathrm{kg} . \mathrm{m}^{-3}\right), N_{A}=6.02283 \times 10^{23}$ the Avogadro number and $\tilde{M}$ the molar mass of the component $\left(\mathrm{kg} \cdot \mathrm{mole}^{-1}\right)$
} 


\section{Hamaker approach : calculation}

We consider the system hydrate/water/hydrate and calculate its Hamaker constant. The use of formulae (4) and (5) requires the knowledge of Van der Waals constants for water/vacuum/water but also water/vacuum/hydrate and hydrate/vacuum/hydrate as well as the corresponding molecular densities. For the sake of simplicity, we introduce the notion of hydrate "molecule" which structure is known (structure sI or sII) and composition equally (46 water molecules and 8 gas molecules for sI and, respectively, 136 and 24 for sII).

We begin to study two hydrate molecules (1) and (2) in the vaccum, separated by a distance $L$. Let us consider a molecule $A$ of water or gas in the molecule (1). All other molecules $I$ of (1) and all other molecules $J$ of (2) each exert on $A$ a force deriving from the Van der Waals interaction potential. The total force that acts on molecule $A$ is then

$$
\vec{F}_{A}=\sum_{I \in(1)-A} \vec{F}_{A I}+\sum_{J \in(2)} \vec{F}_{A J}
$$

So, the hydrate molecule (1) is submitted to an attractive force from (2)

$$
\vec{F}=\sum_{A \in(1)} \vec{F}_{A}
$$

We shall note here that the half of the terms vanishes since the sum of the forces exerted by molecules of (1) on molecules on (1) is zero (internal forces). Consequently,

$$
\vec{F}=\sum_{A \in(1)} \sum_{J \in(2)} \vec{F}_{A J}
$$

Given the interaction potential between two molecules $A$ and $J$, separated by a distance $r, u_{A J(r)}=\frac{-C_{A J J}}{r^{6}}$, we can calculate the force exerted by $J$ on $A$ :

$$
\vec{F}_{A J(r)}=\frac{-\partial u_{A J(r)}}{\partial r} \cdot \frac{\overrightarrow{A J}}{\|\overrightarrow{A J}\|}=\frac{-6 . C_{A J}}{\|\overrightarrow{A J}\|^{7}} \cdot \frac{\overrightarrow{A J}}{\|\overrightarrow{A J}\|}
$$

The calculations have been performed numerically using data in table 3 and make it possible to compute the intensity of the attractive force between two hydrate molecules as a function of their separation distance. From that, we can deduce the interaction potential and, by formal analogy with the equation (1), find the Van der Waals constant $C_{h h}$. In fact, this "constant" varies with distance and tends to a finite limit $\bar{C}_{h h}=3.500 \times 10^{-74} \mathrm{~J} \mathrm{~m}^{6}$ for long ${ }^{3}$ distances (above $30 \mathrm{~nm}$ ). The figure 1 represents the Van der Waals constant $C_{h h}$ as a function of the center-to-center distance of two hydrate molecules ${ }^{4}$. When $d=1.5 \mathrm{~nm}$, we can see that it is 20 times bigger than its asymptotic value.

We can also calculate the Van der Waals constant between an hydrate molecule and a water molecule. For long distances, this constant tends to

\footnotetext{
${ }^{3}$ These distances are to be compared with the hydrate lattice parameter which is $1.203 \mathrm{~nm}$ for sI hydrates.

${ }^{4}$ The center of an hydrate molecule is the position of a given water molecule that constitute it. Refer to the description of the hydrate crystalline structure by Stackelberg [14].
} 
$\bar{C}_{h w}=6.930 \times 10^{-76} \mathrm{~J} . \mathrm{m}^{6}$. For short distances, the figure 2 represents its variation. When the distance is above $1 \mathrm{~nm}$, the value of $C_{h w}$ does not differ from its asymptotic value by more than $38 \%$.

We can now calculate the Hamaker constant for hydrates in vacuum. With the asymptotic values, we have

$$
A_{h h}^{H}=\pi^{2} \cdot C_{h h} \cdot \rho_{h}^{2}
$$

where $\rho_{h}$ is the hydrate molecular density. Since the methane hydrate lattice parameter is $1.203 \mathrm{~nm}$, the density is $912.6 \mathrm{~kg} / \mathrm{m}^{3}$ and the molecular density $\rho_{h}$ is equal to $5.744 \times 10^{26} \mathrm{~m}^{-3}$. Consequently, the Hamaker constant is $1.140 \times$ $10^{-19} \mathrm{~J}$ or ${ }^{5}$ approximatively $30.2 \mathrm{kT}$.

When vacuum is replaced by liquid water, the Hamaker constant shall be calculated by

$$
A_{h w h}^{H}=A_{h h}^{H}+A_{w w}^{H}-2 A_{h w}^{H}
$$

The constant $A_{h w}^{H}$ is equal to $A_{h w}^{H}=\pi^{2} . C_{h w} \cdot \rho_{h} . \rho_{w}$ that is about $1.3130 \times 10^{-19}$ $\mathrm{J}$ with a molecular density of $\rho_{w}=3.342 \times 10^{28}$ molecules $/ \mathrm{m}^{3}$ for water. The constant $A_{w w}^{H}$ is equal to $A_{w w}^{H}=\pi^{2} \cdot C_{w w} . \rho_{w}^{2}$ or $1.5322 \times 10^{-19} \mathrm{~J}$. Finally, the Hamaker constant of the hydrate/water/hydrate system is :

$$
A_{h w h}^{H}=4.589 \times 10^{-21} \mathrm{~J} \approx 1.217 \mathrm{kT}
$$

In the hypothetical case of an hydrate without host gas (methane gas in our case), we would have $\bar{C}_{h w}=6.394 \times 10^{-76}$ and $\bar{C}_{h h}=2.9412 \times 10^{-74} \mathrm{~J}^{-\mathrm{m}^{6}}$ and then, the constant would be $A_{h w h}^{H}=3.928 \times 10^{-20}$ J. Noting $\theta$ the occupancy rate of the hydrate cages, the Hamaker constant can be written

$$
A=[3.928-3.469 \theta] \times 10^{-20} \mathrm{~J}
$$

\section{Lifshitz approach : theory}

\subsection{Notations}

The Hamaker method suffers from two drawbacks : the additivity assumption is strong and the retardation effect is not taken into account. That is the reason why Lifshitz developed an other theory, called macroscopic theory. This theory was then extended in 1961 by Dzyaloshinskii, Lifshitz and Pitaevskii [15] (known as "DLP" theory). This theory considers the medium as continuous and characterized by interconnected parameters

- the refractive index $n_{(w)}$ and the absorption coefficient $k_{(w)}$ (both real). The latter measures the fraction of the wave energy that will be dissipated in heat; it is zero for transparent materials

- the complex index of refraction $N_{(w)}=n_{(w)}+i . k_{(w)}$

- the complex dielectric constant $\epsilon_{(\omega)}$ such that $\epsilon_{(\omega)}=N_{(w)}^{2}$

\footnotetext{
${ }^{5}$ Hamaker constants are sometimes expressed in multiples of $\mathrm{kT}$. At $0^{\circ} \mathrm{C}$, the $k T$ product is $3.7708 \times 10^{-21} \mathrm{~J}$.
} 
- the real part $\epsilon_{(\omega)}^{\prime}$ and imaginary part $\epsilon_{(\omega)}^{\prime \prime}$ of the complex dielectric constant $\epsilon_{(\omega)}=\epsilon_{(\omega)}^{\prime}+i \cdot \epsilon_{(\omega)}^{\prime \prime}$

The complex dielectric constant $\epsilon_{(\omega)}$ of a continuous medium is the linear response, in terms of electric displacement, to an electromagnetic field oscillating at the pulsation $\omega^{6}$. It contains all information on translational, rotational and vibrational relaxations and on the electronic relaxations of the molecules of the considered medium. The imaginary part $\epsilon_{(\omega)}^{\prime \prime}$ is always positive and represents the absorption of the medium, that is to say the energy dissipation of a propagating wave. The graphical representation of $\epsilon_{(\omega)}^{\prime \prime}$ is called the absorption spectrum. The real part $\epsilon_{(\omega)}^{\prime}$ quantifies transmission properties of the medium. Its graphical representation is called transmission spectrum. When $\omega=0$, the complex dielectric constant is equal to the static dielectric constant $\epsilon_{0}$.

In the theory of linear response [16], the definition of the complex dielectric constant $\epsilon$ can be extended to complex variables. For $\omega$ in the complex ensemble, $\epsilon(-\bar{\omega})=\overline{\epsilon(\omega)}$ where the bar symbolizes the conjugated complex. For the purely imaginary pulsation $i \omega$, this relationship implies $\epsilon_{(i \omega)}=\overline{\epsilon_{(i \omega)}}$ and consequently that the imaginary part of $\epsilon_{(i \omega)}=\epsilon_{(i \omega)}^{\prime}+i . \epsilon_{(i \omega)}^{\prime \prime}$ is zero. Hence, $\epsilon_{(i \omega)}=\epsilon_{(i \omega)}^{\prime}$. This shows the reality of $\epsilon_{(i \omega)}$ that is called dielectric response function. Its knowledge is indispensable to the application of the Lifshitz theory.

\subsection{Hamaker constant according to Lifshitz}

The Lifshitz theory is complete and exact in that it takes into account the multi-body interactions and the retardation effect. However, its complexity prevents any application to complex geometries. In what follows, we will study the particular case of two parallel semi-infinite plates separated by a very small distance $d$.

We have previously seen in equation (3) that the Hamaker method leads to an approximated expression of the interaction potential ${ }^{7}$

$$
U_{(d)}^{H}=-A^{H} \cdot f_{(d, g e o m e t r y)}
$$

The Lifshitz theory gives the exact expression of this potential. We call Hamaker constant in the sense of Lifshitz the quantity $A^{L}$ such that the exact potential $U$ is

$$
U_{(d)}=-A^{L} \cdot f_{(d, g e o m e t r y)}
$$

where $f$ is the above defined function. Rigorously, $A^{L}$ depends on the distance $d$. This is thus not a constant and, for that reason, it is sometimes called Hamaker coefficient. However, when distance $d$ is very small, this dependence disappears and $A^{L}$ tends to a value $A^{L 0}$ that we call non retarded Hamaker constant in the sense of Lifshitz.

The coefficient $A_{(d)}^{L}$ can be calculated with the following equations as well as its limit value $A^{L 0}$. Using the field quantum theory and the Feynman diagrams

\footnotetext{
${ }^{6}$ The pulsation $\omega$, the frequency $\nu$ and the wavelength $\lambda$ are connected by the relations $\omega=2 \pi \nu$ and $\lambda=\frac{c}{\nu}$.

${ }^{7}$ In our geometrical configuration, the function $f$ is $1 /\left(12 \pi d^{2}\right)$
} 
technique, Dzyaloshinskii, Lifshitz and Pitaevski proposed the following formula

$$
A_{(d)}^{L}=\frac{-3 k T}{2} \sum_{n=0}^{\infty}, \int_{r_{n}}^{\infty} x \cdot \ln D_{(x)} \cdot d x
$$

where the prime on the sum means that the term $n=0$ has to be multiplied by $\frac{1}{2}$. The function $D_{(x)}$ is equal to

$$
\begin{gathered}
D_{(x)}=\left(1-\bar{\Delta}_{13} \cdot \bar{\Delta}_{23} \cdot e^{-x}\right) \cdot\left(1-\Delta_{13} \cdot \Delta_{23} \cdot e^{-x}\right) \\
\bar{\Delta}_{j 3}=\frac{x \cdot \epsilon_{j\left(i \omega_{n}\right)}-x_{j} \cdot \epsilon_{3\left(i \omega_{n}\right)}}{x \cdot \epsilon_{j\left(i \omega_{n}\right)}+x_{j} \cdot \epsilon_{3\left(i \omega_{n}\right)}} \\
\Delta_{j 3}=\frac{x-x_{j}}{x+x_{j}} \\
x_{j}=\sqrt{x^{2}-\left(1-\frac{\epsilon_{j\left(i \omega_{n}\right)}}{\epsilon_{3\left(i \omega_{n}\right)}}\right) \cdot r_{n}^{2}} \\
r_{n}=2 d \cdot \frac{2 \pi k T \cdot n}{\hbar \cdot c} \cdot \sqrt{\epsilon_{3\left(i \omega_{n}\right)}} \\
\omega_{n}=\frac{2 \pi k T}{\hbar} \cdot n
\end{gathered}
$$

When the distance $d$ is very small, all the $r_{n}$ terms can be approximated by zero. Consequently, $x_{1}=x_{2}=x$ and $\Delta_{13}=\Delta_{23}=0$. The Hamaker constant becomes

$$
A^{L 0}=\frac{-3 k T}{2} \sum_{n=0}^{\infty}, \int_{0}^{\infty} x \cdot \ln D_{(x)} \cdot d x
$$

where the function $D$ is now

$$
\begin{gathered}
D_{(x)}=1-\Delta_{13} \cdot \Delta_{23} \cdot e^{-x} \\
\Delta_{j 3}=\frac{\epsilon_{j\left(i \omega_{n}\right)}-\epsilon_{3\left(i \omega_{n}\right)}}{\epsilon_{j\left(i \omega_{n}\right)}+\epsilon_{3\left(i \omega_{n}\right)}}
\end{gathered}
$$

As noticed by Hough \& White [17], the function $D$ is comprised between 0 and 1 strictly. So, it is possible to develop this term in a series of powers and integrate it term by term to obtain

$$
A^{L 0}=\frac{3 k T}{2} \sum_{n=0}^{\infty}, \sum_{s=1}^{\infty} \frac{\left(\Delta_{13} \cdot \Delta_{23}\right)^{s}}{s^{3}}
$$

In case of truncation of the sum on $s$ just after the first term, the error is below $15 \%$ since $\Delta_{13} . \Delta_{23}<1$. In general, this error is even lower than $5 \%$. For this reason, the following expression is often written

$$
A^{L 0} \approx \frac{3 k T}{2} \sum_{n=0}^{\infty}{ }^{\prime} \Delta_{13} \cdot \Delta_{23}
$$

However, the use of the computer is anyway required and it is not more difficult to entirely calculate the sum on $s$. 
Because of the sampling (summation on $n$ ), the knowledge of the permittivities is not necessary on the whole spectrum. Notably, their evolution within the frequency range comprised between 0 and $\nu_{1}=3.578 \times 10^{13} \mathrm{~s}^{-1}$ at $0^{\circ} \mathrm{C}^{8}$ does not play any role on the interaction potential. More precisely, the main contributions come from the zero-frequency term and the ultra-violet range (between 280 and $400 \mathrm{~nm})$.

\section{Lifshitz approach : calculation}

\subsection{Dielectric data for basic materials}

To calculate the Hamaker constant according to the Lifshitz theory, we need the expression of the dielectric response function and the static dielectric constant for the following materials : methane gas molecules, liquid water molecules and water molecules in hydrate structure. For the latter, we will use the dielectric response function of water molecules in ice structure, as will be justified in section 5.2.1. Additionnally, we give the dielectric data for quartz and dodecane.

\subsubsection{Liquid water}

The dielectric response function $\epsilon_{3(i \omega)}$ of liquid water, which is the intermediate medium in our geometric configuration, can be calculated by different methods. We use here a common way, based on a parametric representation of the dielectric behaviour of materials, using a model of damped oscillators proposed by Ninham \& Parsegian [18]

$$
\epsilon_{(\omega)}=1+\frac{d}{1-i . \hbar \omega \cdot \tau}+\sum_{j} \frac{f_{j}}{\omega_{j}^{2}-i . g_{j} \hbar \omega-(\hbar \omega)^{2}}
$$

The second term of the sum, called Debye relaxation term, models the molecule behaviour in micro-waves. The relaxation time is noted $\tau$ and the oscillator strength $d$. In the third term of the sum $, f_{j}, \omega_{j}$ and $g_{j}$ represent respectively the oscillator strength, its resonance frequency and its bandwidth. This equation 9 is valid for any number, real or complex, and in particular for the purely imaginary pulsation $i \omega$

$$
\epsilon_{(i \omega)}=1+\frac{d}{1+\hbar \omega \cdot \tau}+\sum_{j} \frac{f_{j}}{\omega_{j}^{2}+g_{j} \hbar \omega+(\hbar \omega)^{2}}
$$

Parameters for water, consigned in the table 4, are given by Parsegian [19] and modified by Roth \& Lenhoff [20].

Concerning the static dielectric constant of liquid water, Klein \& Swift [21] proposed the correlation $\epsilon_{0}=88.045-0.4147 T+6.295 \times 10^{-4} T^{2}+1.075 \times 10^{-5} T^{3}$

\footnotetext{
${ }^{8}$ It corresponds to a pulsation lower than $2.248 \times 10^{14}$ rad.s ${ }^{-1}$ or a wavelength greater than $8.385 \mu \mathrm{m}$ or a photon energy below $0.1479 \mathrm{eV}$. The constant $\frac{\hbar}{1 \mathrm{eV}}$ is equal to $6.5789 \times 10^{-16}$.

${ }^{9}$ The result of the formula is correct when the parameters $\left(\omega_{j}, f_{j}, g_{j}\right)$ are expressed in Joule $\left(1 \mathrm{eV}=1.60252 \times 10^{-19} \mathrm{~J}\right)$ and the pulsation in rad.s ${ }^{-1}\left(\hbar=1.0543 \times 10^{-34} \mathrm{~J} . s . r a d^{-1}\right)$
} 
where the temperature $\mathrm{T}$ is in Celsius. In what follows, we will take the value $\epsilon_{30}=88.045$. The dielectric response function for liquid water is displayed in the figure 5 .

\subsubsection{Free gas}

For the molecule of free gas (f), we use the classical model of a simple harmonic oscillator with an absorption frequency $\nu_{e}=\frac{\omega_{e}}{2 \pi}$, which means that the gas has only one absorption line. We have then

$$
\epsilon_{f(\omega)}=1+\frac{n^{2}-1}{1-\left(\frac{\omega}{\omega_{e}}\right)^{2}}
$$

where $n$ is the refractive index in the visible range, approximately equal to $\sqrt{\left.\epsilon_{\left(\omega_{v i s}\right)}\right)}$. Hence, the dielectric response function is

$$
\epsilon_{f(i \omega)}=1+\frac{n^{2}-1}{1+\left(\frac{\omega}{\omega_{e}}\right)^{2}}
$$

According to Israelachvili [7], the absorption frequency is about $\nu_{e}=3 \times 10^{15}$ $\mathrm{Hz}$ that is a pulsation $\omega_{e}=1.885 \times 10^{16} \mathrm{rad} . \mathrm{s}^{-1}$. For methane, the index of refraction in the visible range is $n=1.000444$ [22]. The static dielectric constant of free methane is $\epsilon_{f 0}=1.000944$ at $0^{\circ} \mathrm{C}$ and $760 \mathrm{~mm} \mathrm{Hg}$ [22].

\subsubsection{Ice : parametric method}

Elbaum \& Schick [23] have used experimental data of Kislovskii [24], Daniels [25] and Seki [26] to propose two parametric representations of the ice dielectric constant. The parameters given in the table 5 correspond to the formula

$$
\epsilon_{(\omega)}=1+\sum_{j} \frac{f_{j}}{\omega_{j}^{2}-i \hbar \omega g_{j}-(\hbar \omega)^{2}}
$$

In the following, by Elbaum-Seki's representation, we will mean the parametric representation proposed by Elbaum \& Schick using Kislovskii's data [24] for IR-range and Seki's ones for UV-range [26]. At $0^{\circ} \mathrm{C}$, ice is slightly birefringent; its static dielectric constant is equal to 106.4 along the c-axis and to 91.6 perpendicularly to the latter [7]. The average is $\frac{1}{3}\left(\epsilon_{\|}+2 \epsilon_{\perp}\right)=96.5$. In what follows, we take the value $\epsilon_{i 0}=96.5$.

\subsubsection{Ice : direct calculation}

The parametric representation of the complex dielectric constant is based on an oscillators model. This model is sometimes abusively simple and can lead to inaccurate representation of the material properties. Since the final result is very sensitive to the precision of these data, we will use another way. So, we hope, we will introduce as few errors as possible between the experimental data (ice complex refraction index compiled by Warren [27] over a broad wavelength spectrum) and the final output we want, that is to say the complex dielectric constant for imaginary frequencies. 
Kramers-Kronig relationship Generally speaking, the imaginary and real parts of a response function are linked by the Kramers-Kronig transformations. This transformation has a mathematical origin and hence is not an approximation but a strict equality. It states

$$
\begin{gathered}
\epsilon_{(\omega)}^{\prime}=1+\frac{2}{\pi} \cdot \int_{0}^{\infty} \frac{x \cdot \epsilon_{(x)}^{\prime \prime}}{x^{2}-\omega^{2}} \cdot d x \\
\epsilon_{(\omega)}^{\prime \prime}=\frac{-2 \omega}{\pi} \cdot \int_{0}^{\infty} \frac{\epsilon_{(x)}^{\prime}-1}{x^{2}-\omega^{2}} \cdot d x
\end{gathered}
$$

On the other side, Landau \& Lifshitz [28] have established an other relation that looks similar

$$
\epsilon_{(i \omega)}=1+\frac{2}{\pi} \cdot \int_{0}^{\infty} \frac{x \cdot \epsilon_{(x)}^{\prime \prime}}{x^{2}+\omega^{2}} \cdot d x
$$

We shall note here that the integrals are not ordinary ones. It is the principal part of Cauchy integral, which means that the discontinuity in $\omega$ is circumvented in the integration. The symbol $\int_{0}^{\infty} d x$ used for the sake of simplicity should, rigorously, be replaced by $\lim _{\delta \rightarrow 0}\left(\int_{0}^{\omega-\delta} d x+\int_{\omega+\delta}^{+\infty} d x\right)$. Practically, $\delta$ must be above $10^{2} \mathrm{rad} / \mathrm{s}$ to avoid convergence problems and below $10^{8} \mathrm{rad} / \mathrm{s}$ to ensure an accurate result. In what follows, we take $\delta=10^{5} \mathrm{rad} / \mathrm{s}$.

Adaptation of the Kramers-Kronig formula Practically, the experimental data of the ice refractive index of Warren [27] are not sufficient to calculate $\epsilon_{(x)}^{\prime \prime}$ on the whole spectrum but only in the interval $\Omega_{1}=\left[\omega_{1} ; \omega_{4}\right]$. We may write

$$
\epsilon_{(\omega)}^{\prime}=1+\frac{2}{\pi} \cdot\left(\int_{0}^{\omega_{1}} \frac{x \cdot \epsilon_{(x)}^{\prime \prime}}{x^{2}-\omega^{2}} \cdot d x+\int_{\omega_{1}}^{\omega_{4}} \frac{x \cdot \epsilon_{(x)}^{\prime \prime}}{x^{2}-\omega^{2}} \cdot d x+\int_{\omega_{4}}^{\infty} \frac{x \cdot \epsilon_{(x)}^{\prime \prime}}{x^{2}-\omega^{2}} \cdot d x\right)
$$

Let us imagine that we want to know $\epsilon_{(\omega)}^{\prime}$ for $\omega$ in the interval $\Omega_{2}=\left[\omega_{2} ; \omega_{3}\right] \subset \Omega_{1}$ such that $\left(\frac{\omega_{2}}{\omega_{1}}\right)^{2}$ is very high compared to 1 and $\left(\frac{\omega_{3}}{\omega_{4}}\right)^{2}$ is very small compared to 1 . In this case, the first and third integrals may be approximated and we have

$$
\epsilon_{(\omega)}^{\prime} \approx 1+\frac{2}{\pi} \cdot\left(\frac{-K_{\left(\omega_{1}\right)}}{\omega^{2}}+I_{(\omega)}+Q_{\left(\omega_{4}\right)}\right)
$$

where $K$ and $Q$ are defined by $K_{(t)}=\int_{0}^{t} x \cdot \epsilon_{(x)}^{\prime \prime} \cdot d x$ and $Q_{(t)}=\int_{t}^{\infty} \frac{\epsilon_{(x)}^{\prime \prime}}{x} \cdot d x$. Since $\omega_{1}$ and $\omega_{4}$ are known, $K_{\left(\omega_{1}\right)}$ and $Q_{\left(\omega_{4}\right)}$ are two constants . The integral $I$ is equal to

$$
I_{(\omega)}=\int_{\omega_{1}}^{\omega_{4}} \frac{x \cdot \epsilon_{(x)}^{\prime \prime}}{x^{2}-\omega^{2}} \cdot d x
$$

The figure 3 represents the residual part $R_{(\omega)}=\frac{\pi}{2}\left(\epsilon_{(\omega)}^{\prime}-1\right)-I_{(\omega)}$ calculated directly from Warren data for 200 values of $\omega \in \Omega_{2}=\left[\omega_{2} ; \omega_{3}\right]=\left[\omega_{1} . D ; \omega_{4} / D\right]$. The calculations have been performed with $D=50$. No obvious dependence to $\omega$ can be observed. We deduce that $\frac{K_{\left(\omega_{1}\right)}}{\omega^{2}}$ is negligible compared to $Q_{\left(\omega_{4}\right)}$. In what follows, we take $K_{\left(\omega_{1}\right)}=0$ and $Q_{\left(\omega_{4}\right)}=0.07867$. 
We can now evaluate the dielectric constant on the imaginary axis $\epsilon_{(i \omega)}$. For that, we write

$$
\epsilon_{(i \omega)}=1+\frac{2}{\pi} \cdot\left(\int_{0}^{\omega_{1}} \frac{x \cdot \epsilon_{(x)}^{\prime \prime}}{x^{2}+\omega^{2}} \cdot d x+\int_{\omega_{1}}^{\omega_{4}} \frac{x \cdot \epsilon_{(x)}^{\prime \prime}}{x^{2}+\omega^{2}} \cdot d x+\int_{\omega_{4}}^{\infty} \frac{x \cdot \epsilon_{(x)}^{\prime \prime}}{x^{2}+\omega^{2}} \cdot d x\right)
$$

and, as it has been done above, give the approximation

$$
\epsilon_{(i \omega)} \approx 1+\frac{2}{\pi} \cdot\left(\frac{K_{\left(\omega_{1}\right)}}{\omega^{2}}+\int_{\omega_{1}}^{\omega_{4}} \frac{x \cdot \epsilon_{(x)}^{\prime \prime}}{x^{2}+\omega^{2}} \cdot d x+Q_{\left(\omega_{4}\right)}\right) \approx 1+\frac{2}{\pi} \cdot\left(J_{(\omega)}+Q_{\left(\omega_{4}\right)}\right)
$$

where the integral $J$ is defined by the relation

$$
J_{(\omega)}=\int_{\omega_{1}}^{\omega_{4}} \frac{x \cdot \epsilon_{(x)}^{\prime \prime}}{x^{2}+\omega^{2}} \cdot d x
$$

As illustrated on the figure 4, the calculation on the frequency domain of interest gives a result very close to that obtained with the Elbaum-Seki parametric representation [23].

The dielectric response function of ice is compared to liquid water one in the figure 5 .

\subsubsection{Other materials}

For other materials, like quartz and dodecane, Hough \& White [17] proposed the following representation

$$
\epsilon_{(i \omega)}=1+\frac{C_{I R}}{1+\left(\frac{\omega}{\omega_{I R}}\right)^{2}}+\frac{C_{U V}}{1+\left(\frac{\omega}{\omega_{U V}}\right)^{2}}
$$

where the parameters are given in the table 6 . The pulsations $\omega_{I R}$ and $\omega_{U V}$ are expressed in $\mathrm{rad} / \mathrm{s}$.

\subsection{Dielectric data for hydrate}

\subsubsection{Dielectric response function}

To calculate the hydrate dielectric response function, we will do the following three assumptions

1. The hydrate (1) is considered as the superposition of a water structure (e) and of gas (g). For any frequency, its dielectric permittivity $\epsilon_{1}$ is given by the mixing rule of Clausius-Mosotti that expresses the additivity of the total polarisabilities

$$
\frac{\epsilon_{1}-1}{\epsilon_{1}+2}=\frac{\epsilon_{e}-1}{\epsilon_{e}+2}+\frac{\epsilon_{g}-1}{\epsilon_{g}+2}
$$


2. A water molecule (e) in the hydrate crystalline lattice has the same polarisability ${ }^{10}$ as a water molecule in the ice crystalline lattice (i). The Clausius-Mosotti formula states that

$$
\frac{\alpha}{4 \pi \epsilon_{0}}=\frac{\epsilon-1}{\epsilon+2} \cdot \frac{3 V}{4 \pi}
$$

Noting $\epsilon_{i}$ the dielectric constant of the ice (i), we have

$$
\frac{\epsilon_{e}-1}{\epsilon_{e}+2}=\frac{\epsilon_{i}-1}{\epsilon_{i}+2} \cdot \frac{\rho_{e}}{\rho_{i}}
$$

3. A gas molecule (g) in a hydrate cavity has the same polarisability as a free gas molecule (f) at atmospheric pressure and $0^{\circ} \mathrm{C}$. Thanks to ClausiusMosotti formula, we have

$$
\frac{\epsilon_{g}-1}{\epsilon_{g}+2}=\frac{\epsilon_{f}-1}{\epsilon_{f}+2} \cdot \frac{\rho_{g}}{\rho_{f}}
$$

The sum of the two contributions allows us to calculate the hydrate dielectric response function. We can give an interpolation of this result. For $\omega \in\left[2.248 \times 10^{14} ; 4.255 \times 10^{16}\right]{\mathrm{rad} . \mathrm{s}^{-1}}$, it is correctly approximated by

$$
\epsilon_{1(i \omega)}=\sum_{n=0}^{8} a_{n} \cdot \log _{10}^{n}(\hbar \omega)
$$

The coefficients are in the table 7

The figure 5 gives a graphical summary of the dielectric response functions of liquid water, ice and hydrate.

\subsubsection{Static dielectric constant}

For water ice, the density is $\rho_{i}=917 \mathrm{~kg} . \mathrm{m}^{-3}$. The water density in hydrate being $\rho_{e}=\frac{46 \times 18 \times 10^{-3}}{N_{A} \cdot\left(12.03 \times 10^{-10}\right)^{3}}=790.6 \mathrm{~kg} \cdot \mathrm{m}^{-3}$, we have $\epsilon_{e 0}=16.29$ for the static dielectric constant of water in hydrate. In the same logic, for gas, with $\rho_{g}=\frac{8 \times 16 \times 10^{-3}}{N_{A} \cdot\left(12.03 \times 10^{-10}\right)^{3}}=122.1 \mathrm{~kg} \cdot \mathrm{m}^{-3}$ and $\rho_{f}=\frac{P \tilde{M}}{R T}=0.7139 \mathrm{~kg} \cdot \mathrm{m}^{-3}$, we have $\epsilon_{g 0}=1.171$ for the static dielectric constant of gas (g) in hydrate. Consequently, with the help of the mixing rule given above, we can calculate the hydrate static dielectric constant $\epsilon_{10}=17.46$.

\subsection{Hamaker constant}

With all these data, we performed the numerical computations and found the Hamaker constant to be $A^{L 0}=4.8647 \times 10^{-20} \mathrm{~J}$ when the intermediate medium is vacuum and $A^{L 0}=8.2520 \times 10^{-21} \mathrm{~J}$ when it is liquid water. Expressed in multiples of $\mathrm{kT}$, we obtain respectively 12.90 and $2.188 \mathrm{kT}$. Finally, the non retarded Hamaker constant in the sense of Lifshitz is

$$
A_{h w h}^{L 0}=8.2520 \times 10^{-21} J \approx 2.188 k T
$$

\footnotetext{
${ }^{10}$ Polarisability means total polarisability (electronic and orientation)
} 


\section{Hybrid approach : theory}

\subsection{General philosophy}

As already mentioned, the full application of the DLP theory has two advantages (the retardation effect and the multi-body interactions are taken into account) but an analytical expression is available only for simple geometries. Inversely, the application of the Hamaker method is applicable for complex geometries but needs the additivity hypothesis and neglects the retardation effect. To circumvent these problems, we will make an hybridation ${ }^{11}$. The interaction potential will be approximated by

$$
\bar{U}_{(d)}=A^{L 0} \cdot f_{(d, \text { geometry })} \cdot \Gamma_{(d, g e o m e t r y)}
$$

We must give some comments on this hybridation.

- The function $f_{(d, \text { geometry })}$ is calculated with the Hamaker approach. The table 9 shows its value for a given geometrical configuration. For other geometries, even complex ones, its value can be easily calculated (Cf. Tadmor [10]).

- The constant $A^{L 0}$ is the non retarded Hamaker constant in the sense of Lifshitz, computed in the case of two parallel, semi-infinite plates. This way, the multi-body interactions and the presence of the intermediate (liquid water in our case) are taken into account.

- The function $\Gamma_{(\text {d,geometry })}$ expresses the retardation effect. When the distance tends to zero, this function $\Gamma$ tends to 1 .

\subsection{Retardation effect}

To take into account the retardation effect in this hybrid approach, it is usual to modify the London equation (1). So, the interaction potential between two molecules takes the form $u_{(r)}=-\frac{C}{r^{6}} \cdot g_{(r)}$. The function $g$ tends to 1 when the intermolecular distance $r$ tends to zero. The literature gives many expressions for the corrective function $g_{(r)}$. For two infinite half-spaces, we can obtain the exact expression using the Lifshitz theory and a formal analogy. It is the same for two half spheres (Cf. Langbein [29]). These expressions are however complicated and various approximations have been proposed : Casimir \& Polder [6] used quantum electrodynamics to find the exact expression for two identical neutral atoms where only one excited state contributes to the London energy. It is the most accurate expression. Overbeek [30] simplified this expression and gave piece-wise functions on two domains. Schenkel \& Kitchener [31] proposed a unique function for the range $\lambda \geq 8 \mathrm{~nm}$. Anandarajah \& Chen [32] proposed a unique function valid between 0 and $1000 \mathrm{~nm}$. These authors used then these corrective functions $g$ to calculate the $\Gamma$ function for different geometries.

${ }^{11}$ The method and the expression is from Parsegian [19] 


\subsubsection{Two parallel semi-infinite plates}

For two parallel semi-infinite plates separated by a distance $d$, Overbeek [33] used his function $g$ to find the retarded interaction potential d'

$$
\begin{gathered}
\Gamma_{(d)}^{o}=1.01-1.12 a d+0.696 a^{2} d^{2}-0.229 a^{3} d^{3}+0.031 a^{4} d^{4} \\
\Gamma_{(d)}^{o}=\frac{0.490}{a d}-\frac{0.102}{a^{2} d^{2}}
\end{gathered}
$$

respectively for $d \leq \frac{3}{2 a}$ and for $d>\frac{3}{2 a}$. The constant $a$ is equal to $a=\frac{\pi}{\lambda}$ with $\lambda=100 \mathrm{~nm}$. Anandarajah \& Chen [32] used their own corrective function $g$ and came to the expression :

$$
\Gamma_{(d)}^{a c}=1-\frac{4 d}{c}-\frac{18 d^{2}}{c^{2}}-\frac{12 d^{3}}{c^{3}}+\frac{12 d^{2}}{c^{4}} \cdot(d+c)^{2} \cdot \ln \left(1+\frac{c}{d}\right)
$$

$c=\frac{b \lambda}{2 \pi}$ with $\lambda=100 \mathrm{~nm}$ and $b=3.1$.

\subsubsection{A sphere and a semi-infinite plate}

A sphere of radius $r$, separated by a distance $d$ of a semi-infinite plate, is submitted to an interaction potential given in the equations $(7),(8)$ and (9) of Chen \& Anandarajah [34], who use their corrective function $g$.

\subsubsection{Two spheres}

Two spheres of radius $r_{1}$ and $r_{2}$, which centers are at a distance $h$ are submitted to an interaction potential given in the equations (18), (19) and (20) of Chen \& Anandarajah [34]. In their article, this expression is compared to the exact expression that Clayfield et al. [35] proposed for two spheres using the corrective function of Casimir \& Polder [6]. Langbein [29] applied the Lifshitz theory to this geometric configuration and obtained the exact solution. However it requires heavy calculations and numerical convergence problems make it difficult to use. The Lifshitz theory can also be applied indirectly using the Derjaguin approximation [36] or the one of Papadopoulos-Cheh [37]. The article of Thennadil [38] proposes a complete review of their calculation methods and their respective advantages.

\section{Hybrid approach : calculation}

As an example for this hybrid approach, we consider two spherical hydrate particles. According Chen \& Anandarajah [34], two spheres of radius $r_{1}$ and $r_{2}$ placed at a center-to-center distance $h$ are submitted to the interaction potential

$$
\begin{gathered}
U(h)=A^{L 0} \cdot\left[U_{0}(h)+U_{1}(h)\right] \\
U_{0}(h)=\frac{-1}{6} \cdot\left[\frac{2 r_{1} r_{2}}{d_{1} d_{3}}+\frac{2 r_{1} r_{2}}{d_{2} d_{4}}+\ln \frac{d_{1} d_{3}}{d_{2} d_{4}}\right]
\end{gathered}
$$




$$
U_{1}(h)=\frac{-1}{30 c^{4} h} \cdot\left[4 c r_{1} r_{2}\left(4 r_{1}^{2}+4 r_{2}^{2}+2 h^{2}+7 h c+9 c^{2}\right)+c^{4}(c+5 h) \ln \frac{d_{2} d_{4}}{d_{1} d_{3}}+\sum_{i=1}^{4} b_{i} \ln \frac{e_{i}}{d_{i}}\right]
$$

with following notations :

$$
\begin{gathered}
d_{1}=h-r_{1}-r_{2} \\
d_{2}=h+r_{1}-r_{2} \\
d_{3}=h+r_{1}+r_{2} \\
d_{4}=h-r_{1}+r_{2} \\
e_{i}=d_{i}+c \\
b_{i}=(-1)^{i+1} e_{i}^{4}\left[4 e_{i}-5(h+c)\right]-20 r_{1} r_{2} e_{i}^{3} \\
c=\frac{b \lambda}{2 \pi}
\end{gathered}
$$

with $\mathrm{b}=3.1$ and $\lambda=100 \mathrm{~nm}$.

The figures 6 and 7 show the absolute value of the interaction potential as well as the attractive force between two equal spheres, when the radius varies.

\section{Discussion}

Concerning hydrate, we find a value of $\epsilon_{10}=17.46$ for its static dielectric constant. This value is $70 \%$ smaller than the value of 58 found experimentally by Makogon [39]. However, these values are both credible since they both are between the static dielectric constant of liquid water (88.045) and of gas (1.171), dodecane (1.991) or quartz (4.29). With Makogon's value, the non retarded Hamaker constant is $A^{L 0}=7.03 \times 10^{-21} \mathrm{~J}$ instead of $8.25 \times 10^{-21} \mathrm{~J}$, that is a difference of only $15 \%$.

We shall cite here the work of Camargo \& Palermo [40] who use Israelachvili's formula [7] to get a first estimate of the Hamaker constant. They found a value of $5.2 \times 10^{-21} \mathrm{~J}$, which is used in Yang's work et al. [41]. Qualitatively, our results valid their estimate a posteriori.

Concerning the ice dielectric response function, which is the base to calculate hydrate's one, we have seen that our result, obtained by application of the Kramers-Kronig transformations, is slightly different from the result obtained using the parametric representation that Elbaum \& Schick [23] built from Seki's data [26]. If we use this dielectric response function, the Hamaker constant would be $A^{L 0}=8.36 \times 10^{-21} \mathrm{~J}$ instead of $8.25 \times 10^{-21} \mathrm{~J}$ that is a difference of the order of $1 \%$. Here again, the robustness of our result seems reassessed.

If we use Makogon's value [39] for the ice static dielectric constant and the parametric representation of Elbaum \& Schick [23] for ice, then the Hamaker constant would be $A^{L 0}=7.13 \times 10^{-21} \mathrm{~J}$ instead of $8.25 \times 10^{-21} \mathrm{~J}$ that is a difference of the order of $14 \%$. 
We can extend our work to systems containing a third material $M$. The table 8 shows the Hamaker constants $A^{L 0}$ for Hydrate-Water-M or HydrateVacuum-M systems. The dielectric response functions $\epsilon_{(i \omega)}$ are from Hough \& White [17] for dodecane, quartz, sapphire and teflon. For the three metals (gold, silver and copper), data are from Parsegian \& Weiss [42].

Finally, we can predict the most probable configurations. For instance, the Hamaker constant of the system Quartz-Hydrate-Water $\left(6.509 \times 10^{-21} \mathrm{~J}\right)$ is higher than that of the system Quartz-Water-Hydrate $\left(2.890 \times 10^{-21} \mathrm{~J}\right)$. It means that quartz attracts more water than hydrate and thus that the stable situation is an hydrate particle separated from quartz by a liquid film. Also, the Hamaker constant of the Hydrate-Dodecane-Water system $\left(10.35 \times 10^{-21}\right.$ $\mathrm{J})$ is positive. It means that, in a water in dodecane emulsion, water droplets will agglomerate with hydrate particles. Inversely, for an dodecane in water emulsion, dodecane droplets will tend to agglomerate to hydrate particles. In this case, we shall notice that the Hamaker constant of the Hydrate-DodecaneWater system $\left(10.35 \times 10^{-21} \mathrm{~J}\right)$ is higher than for the Hydrate-Water -Dodecane system $\left(1.989 \times 10^{-21} \mathrm{~J}\right)$. Consequently, the hydrate attracts more water than dodecane and thus the stable situation is an hydrate particle with a double layer : a water film in contact with the hydrate particle and a dodecane layer on the outside. This situation will happen only if the dodecane surface tension is small enough to allow the droplet-to-shell transition. In the same logic, data from table 8 show that hydrates in suspension in water will aggregate, in order of preference, with metal, sapphire and quartz. Experiment with unconsolidated quartz grains placed in a metallic reactor have to be interpreted with precaution.

\section{Conclusion}

In this article, we give an insight of Van der Waals forces acting in systems involving gas hydrates. The microscopic, Hamaker approach to calculate the Hamaker constant of the hydrate-water-hydrate system leads to a value of $4.59 \times 10^{-21} \mathrm{~J}$ which is close to the value of $8.25 \times 10^{-21} \mathrm{~J}$ found with the macroscopic, Lifshitz theory. To apply the latter, we used the Kramers-Kronig relationship and adapted it to use the full information contained in optical experimental data of ice, since water molecules frozen in a rigid structure appear in both ice and gas hydrate solids. Although the Hamaker constant obtained with the Lifshitz theory appears to us more representative of the reality, the difference between the two values is rather low in terms of agglomeration kinetics. In effect, the characteristic agglomeration time is proportional to the inverse agglomeration efficiency $\alpha$, which is proportional to the Hamaker constant at the power 0.18 [43]. Numerically, this means that the two Hamaker constants lead to a difference of $10 \%$ only between the two characteristic agglomeration times. We then applied this theory to different other systems. Notably, we studied the geometry of two spherical hydrate particles interacting across water and we compared different three-phases systems to predict what configurations would be more stable. In this optic, we applied the theory to systems containing hydrates and water but also ice, dodecane, quartz, sapphire, teflon and metals. These data may appear of interest for industrial and experimental purposes. 


\section{References}

[1] E.D. Sloan, Clathrate Hydrates of Natural Gases, $2^{\text {nd }}$ Ed., Marcel Dekker, New-York, 1997

[2] W.H. Keesom, Physikalische Zeit. 22 (1921) 129

[3] P. Debye, Physikalische Zeit. 22 (1921) 302

[4] R. Eisenschitz \& F. London, Zeit. für Physik 60 (1930) 491

[5] F. London, Trans. Faraday Soc. 33 (1937) 8

[6] H.B.G. Casimir \& D. Polder, Phys. Rev. 73 (1948) 360

[7] J. Israelachvili, Intermolecular and Surface Forces, $2^{\text {nd }}$ Ed., Academic Press, London, 1992

[8] H.C. Hamaker, Physica 4 (1937) 1059

[9] M. Elimelech , J. Gregory, X. Jia \& R. Williams, Particle Deposition and Aggregation, Butterworth \& Heinemann Ltd., Oxford, 1995

[10] R. Tadmor, J. of Physics : Condensed Matter 13 (2001) 195-202

[11] S. Bhattacharjee \& A. Sharma, J. Colloid Interf. Sci., 171 (1995) 288-296

[12] L. Zeman \& M. Wales, in "Synthetic Membranes : HF and UF Uses", A.F. Turbak Ed., ACS Symp. Ser., Vol. 154, p.411, Amer. Chem. Soc., Washington DC, 1981

[13] K.D. Papadopoulos \& C. Kuo, Colloids Surf. 46 (1990) 115

[14] M. von Stackelberg \& H.R. Müller, Feste Gashydrate II. Struktur und Raumchemie, Zeitschrift für Elektrochemie, 58-1, (1954) 25-39

[15] I.E. Dzyaloshinskii, E.M. Lifshitz \& L.P. Pitaevskii, Adv. Phys. 10 (1961) 165-209

[16] L.D. Landau \& E.M. Lifshitz, Statistical Physics, $2^{\text {nd }}$ Ed., Part 1, Pergamon, Oxford, 1980

[17] D.B. Hough \& L.R. White, Adv. Coll. Interf. Sci. 14 (1980) 3-41

[18] V.A. Parsegian \& B.W. Ninham, Nature 224 (1969) 1197

B.W. Ninham \& V.A. Parsegian, Biophys. J. 10 (1970) 646

B.W. Ninham \& V.A. Parsegian, J. Chem. Phys. 52 (1970) 4578

V.A. Parsegian \& B.W. Ninham, Biophys. J. 13 (1973) 209

[19] V.A. Parsegian, Van der Waals Interactions, Physical Chemistry, Theorex, La Jolla, Calif., 1975

[20] C.M. Roth \& A.M. Lenhoff, J. Colloid Interf. Sci. 179 (1996) 637-639

[21] L.A. Klein \& C.T. Swift, IEEE Trans. Antenna and Propagation 25 (1977) $104-111$ 
[22] CRC Handbook of Chemistry and Physics, 68th Ed., CRC Press, Boca Raton, Florida, 1987

[23] M. Elbaum \& M. Schick, Phys. Rev. Letters 66-13 (1991) 1713-1716

[24] L.D. Kislovskii, Opt. Spectroscop. (USSR) 7 (1959) 201 Irvine W.M. \& Pollack J.B., Icarus 8 (1968) 324

[25] J. Daniels, Opt. Commun. 3 (1971) 240

[26] M. Seki, K. Kobayashi \& J. Nakahara, J. Phys. Soc. Jpn. 50 (1981) 2643

[27] S.G. Warren, Applied Optics 23-8 (1984) 1206-1225

[28] L.D. Landau \& E.M. Lifshitz, Electrodynamics of continuous media, Addison-Wesley, Reading, Mass., 1960

[29] D. Langbein, Phys. Rev. B 2 (1970) 3371 D. Langbein, Theory of Van der Waals attraction, Springer Tracts in Modern Physics, Vol. 72, SpringerVerlag, Berlin, New-York, 1974

[30] J. Th. G. Overbeek, in "Colloid Science" (H.R. Kruyt, Ed.), Vol. 1, p.266, Elsevier, Amsterdam, 1952

[31] J.H. Schenkel \& J.A. Kitchener, Trans. Faraday Soc. 56 (1960) 161

[32] A. Anandarajah \& J. Chen, J. Colloid Interf. Sci. 176 (1995) 293-300

[33] J. Th. G. Overbeek, Kon. Ned. Akad. Wetensch. Proc. Se. B 69 (1966) 161

[34] J. Chen \& A. Anandarajah, J. Colloid Interf. Sci. 180 (1996) 519-523

[35] E.J. Clayfield, E.C. Lumb \& P.H. Marckey, J. Colloid Interf. Sci. 37 (1971) 382

[36] B.V. Derjaguin, Kolloid Zeits. 69 (1934) 155-164

[37] K.D. Papadopoulos \& H.Y. Cheh, AIChE J. 30 (1984) 7

[38] S.N. Thennadil \& L.H. Garcia-Rubio, J. Colloid Interf. Sci. 243 (2001) $136-142$

[39] Y.F. Makogon, Hydrates of hydrocarbons, Penn Well Publishing Co., Tulsa, Oklahoma, 1997

[40] R. Camargo \& T. Palermo, Proc. 4th Int. Conf. on Gas Hydrates, Yokohama (2002) 880-885

[41] S. Yang, D.M. Kleehammer, Z. Huo, E.D. Sloan \& K.T. Miller, Micromechanical measurements of hydrate particle attractive forces, 15th Symp. on Thermophysical Properties, Boulder, USA, 2003

[42] V.A. Parsegian \& G.H. Weiss, J. Colloid Interf. Sci. 81-1 (1981) 285-289

[43] T.G.M. Van de Ven \& S.G. Mason, Colloid \& Polymer Sci 255 (1977) 468 


\section{Footnotes}

Note 1 The revolution time of the electron of the Bohr atom is equal to the inverse of the first ionisation frequency which is about $3 \times 10^{15} \mathrm{~s}^{-1}$.

Note 2 The molecular density, expressed in molecules $/ \mathrm{m}^{3}$, is equal to $\rho=$ $\frac{N_{A} \cdot \rho_{V}}{M}$ where $\rho_{V}$ is the density $\left(\mathrm{kg} \cdot \mathrm{m}^{-3}\right), N_{A}=6.02283 \times 10^{23}$ the Avogadro number and $\tilde{M}$ the molar mass of the component $\left(\mathrm{kg} \cdot \mathrm{mole}^{-1}\right)$

Note 3 These distances are to be compared with the hydrate lattice parameter which is $1.203 \mathrm{~nm}$ for sI hydrates.

Note 4 The center of an hydrate molecule is the position of a given water molecule that constitute it. Refer to the descripon of the hydrate crystalline structure by Stackelberg [14].

Note 5 Hamaker constants are sometimes expressed in multiples of kT. At $0^{\circ} \mathrm{C}$, the $k T$ product is $3.7708 \times 10^{-21} \mathrm{~J}$.

Note 6 The pulsation $\omega$, the frequency $\nu$ and the wavelength $\lambda$ are connected by the relations $\omega=2 \pi \nu$ and $\lambda=\frac{c}{\nu}$.

Note 7 In our geometrical configuration, the function $f$ is $1 /\left(12 \pi d^{2}\right)$

Note 8 It corresponds to a pulsation lower than $2.248 \times 10^{14}$ rad.s ${ }^{-1}$ or a wavelength greater than $8.385 \mu \mathrm{m}$ or a photon energy below $0.1479 \mathrm{eV}$. The constant $\frac{\hbar}{1 e V}$ is equal to $6.5789 \times 10^{-16}$.

Note 9 The result of the formula is correct when the parameters $\left(\omega_{j}, f_{j}, g_{j}\right)$ are expressed in Joule $\left(1 \mathrm{eV}=1.60252 \times 10^{-19} \mathrm{~J}\right)$ and the pulsation in rad.s ${ }^{-1}$ $\left(\hbar=1.0543 \times 10^{-34}{\left.\mathrm{~J} . \mathrm{s} . \mathrm{rad}^{-1}\right)}^{-1}\right.$

Note 10 Polarisability means total polarisability (electronic and orientation)

Note 11 The method and the expression is from Parsegian [19] 


\section{Figure legends}

Figure 1 Van der Waals constant between hydrate molecules

Figure 2 Van der Waals constant between an hydrate molecule and a water molecule

Figure 3 Residual part $R_{\omega}$ to calculate the ice dielectric response function

Figure 4 Ice dielectric response function (Elbaum-Seki [23] and this work)

Figure 5 Dielectric response functions of liquid water, ice and hydrate

Figure 6 Interaction potential between two hydrate spheres

Figure 7 Attractive force between two hydrate spheres 


\section{List of Tables}

1 Contributions to the induction, orientation and dispersion inter-

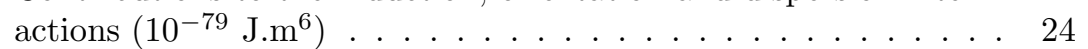

2 Geometric function $f_{(\text {d,geometry })}[9] \ldots \ldots . \ldots . \ldots 25$

3 Hamaker constant for water and hydrocarbons in vacuum . . . . 26

4 Parameters for the dielectric representation of liquid water . . . . 26

5 Parameters for the dielectric representation of ice [23] . . . . . . 27

6 Parameters for the dielectric representation of quartz and dodecane 27

7 Coefficients for the polynomial interpolation of the hydrate dielectric response function . . . . . . . . . . . . . . 28

8 Hamaker constants for different systems involving hydrates $\left(10^{-21}\right.$

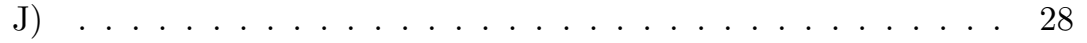




\begin{tabular}{|c|ccc|c|}
\hline & $\mathrm{C}_{\text {ind }}$ & $\mathrm{C}_{\text {orient }}$ & $\mathrm{C}_{\text {disp }}$ & $\mathrm{C}_{\text {total }}$ \\
\hline $\mathrm{H}_{2} \mathrm{O}-\mathrm{H}_{2} \mathrm{O}$ & 10 & 96 & 33 & 139 \\
$\mathrm{CH}_{4}-\mathrm{CH}_{4}$ & 0 & 0 & 102 & 102 \\
$\mathrm{H}_{2} \mathrm{O}-\mathrm{CH}_{4}$ & 9 & 0 & 58 & 67 \\
\hline
\end{tabular}

Table 1: Contributions to the induction, orientation and dispersion interactions $\left(10^{-79}\right.$ J.m $\left.^{6}\right)$ 


\begin{tabular}{ll}
\hline Geometry & $f_{(d, \text { geometry })}$ \\
\hline Two spheres & {$\left[\frac{2 r_{1} r_{2}}{d^{2}+2 r_{1} d+2 r_{2} d}+\frac{2 r_{1} r_{2}}{d^{2}+2 r_{1} d+2 r_{2} d+4 r_{1} r_{2}}+\ln \left(\frac{d^{2}+2 r_{1} d+2 r_{2} d}{d^{2}+2 r_{1} d+2 r_{2} d+4 r_{1} r_{2}}\right)\right] / 6$} \\
Two close spheres & $r_{1} r_{2} /\left[6 d\left(r_{1}+r_{2}\right)\right]$ \\
Sphere - Half space & {$\left[\frac{r}{d}+\frac{r}{d+2 r}+\ln \left(\frac{d}{d+2 r}\right)\right] / 6$} \\
Sphere - close Half space & $r /(6 d)$ \\
Two plates & {$\left[1 / d^{2}+1 /(d+2 e)^{2}-2 /(d+e)^{2}\right] /(12 \pi)$} \\
Two half spaces & $1 /\left(12 \pi d^{2}\right)$ \\
\hline
\end{tabular}

Table 2: Geometric function $f_{(d, g e o m e t r y)}[9]$ 


\begin{tabular}{lccc} 
& $\mathrm{C}$ & $\rho$ & $A_{i i}$ \\
& $\left(10^{-79}{\left.\mathrm{~J} . \mathrm{m}^{6}\right)}^{6}\right.$ & $\left(10^{28} \mathrm{~m}^{-3}\right)$ & $\left(10^{-19} \mathrm{~J}\right)$ \\
\hline Hydrocarbons & 50 & 3.3 & 0.5 \\
Water & 139 & 3.3 & 1.5
\end{tabular}

Table 3: Hamaker constant for water and hydrocarbons in vacuum

\begin{tabular}{ccc}
\hline$\omega_{j}$ & $f_{j}$ & $g_{j}$ \\
$(\mathrm{eV})$ & $\left(\mathrm{eV}^{2}\right)$ & $(\mathrm{eV})$ \\
\hline 8.21 & 3.26 & 0.63 \\
10.0 & 3.87 & 0.84 \\
11.4 & 12.0 & 2.05 \\
13.6 & 63.6 & 3.9 \\
17.8 & 114 & 7.33 \\
25.2 & 24.3 & 5.34 \\
$2.07 \times 10^{-2}$ & $6.25 \times 10^{-4}$ & $1.5 \times 10^{-2}$ \\
$6.9 \times 10^{-2}$ & $3.50 \times 10^{-3}$ & $3.8 \times 10^{-2}$ \\
$9.2 \times 10^{-2}$ & $1.28 \times 10^{-3}$ & $2.8 \times 10^{-2}$ \\
$2.0 \times 10^{-1}$ & $5.69 \times 10^{-3}$ & $2.5 \times 10^{-2}$ \\
$4.2 \times 10^{-1}$ & $1.35 \times 10^{-2}$ & $5.6 \times 10^{-2}$ \\
\hline $\mathrm{d}=74.8$ & & $1 / \tau=1.05 \times 10^{11}{\mathrm{rad} . \mathrm{s}^{-1}}$
\end{tabular}

Table 4: Parameters for the dielectric representation of liquid water 


\begin{tabular}{ccc}
\hline$\omega_{j}$ & $f_{j}$ & $g_{j}$ \\
$(\mathrm{eV})$ & $(\mathrm{eV})$ & $(\mathrm{eV})$ \\
\hline \multicolumn{3}{c}{ IR Parameters [24] } \\
\hline $1.716 \times 10^{-2}$ & $2.110 \times 10^{-3}$ & $8.553 \times 10^{-2}$ \\
0.1140 & $6.754 \times 10^{-3}$ & $5.881 \times 10^{-2}$ \\
0.4003 & $1.089 \times 10^{-2}$ & $2.594 \times 10^{-2}$ \\
\hline \multicolumn{3}{c}{ UV Parameters [25] } \\
\hline 8.740 & 3.081 & 0.7672 \\
11.55 & 11.25 & 2.666 \\
14.49 & 43.12 & 4.374 \\
17.54 & 47.93 & 5.291 \\
21.81 & 41.25 & 8.066 \\
32.83 & 144.8 & 22.80 \\
\hline \multicolumn{3}{c}{0.7308} \\
\hline 8.603 & UV Parameters [26] \\
10.43 & 5.027 & 1.342 \\
12.79 & 5.124 & 1.968 \\
15.06 & 8.885 & 3.701 \\
18.07 & 48.44 & 6.876 \\
22.82 & 54.57 & 15.93 \\
32.97 & 39.92 & \\
\hline
\end{tabular}

Table 5: Parameters for the dielectric representation of ice [23]

\begin{tabular}{lccccc} 
& $C_{I R}$ & $\omega_{I R}$ & $C_{U V}$ & $\omega_{U V}$ & $\epsilon_{0}$ \\
\hline Quartz & 1.93 & $2.093 \times 10^{14}$ & 1.359 & $2.032 \times 10^{16}$ & 4.29 \\
Dodecane & 0.026 & $5.540 \times 10^{14}$ & 0.965 & $0.873 \times 10^{16}$ & 1.991
\end{tabular}

Table 6: Parameters for the dielectric representation of quartz and dodecane 


\begin{tabular}{|c|c||c|c|}
\hline$a_{0}$ & 1.8277 & $a_{5}$ & -0.20779 \\
$a_{1}$ & -0.09573 & $a_{6}$ & 0.11237 \\
$a_{2}$ & 0.07746 & $a_{7}$ & 0.15226 \\
$a_{3}$ & -0.1089 & $a_{8}$ & -0.070614 \\
$a_{4}$ & -0.16035 & & \\
\hline
\end{tabular}

Table 7: Coefficients for the polynomial interpolation of the hydrate dielectric response function

\begin{tabular}{|c|c|c|}
\hline $\mathrm{M}$ & $\mathrm{H}-\mathrm{W}-\mathrm{M}$ & $\mathrm{H}-\mathrm{V}-\mathrm{M}$ \\
\hline Hydrate & 8.252 & 9.460 \\
Dodecane & 1.989 & 0.899 \\
Quartz & 2.890 & 2.780 \\
Teflon & 1.991 & 0.874 \\
Sapphire & 4.506 & 5.329 \\
Gold & 5.018 & 9.711 \\
Copper & 5.029 & 9.722 \\
Silver & 5.031 & 9.724 \\
Ice & 6.975 & 9.923 \\
\hline
\end{tabular}

Table 8: Hamaker constants for different systems involving hydrates $\left(10^{-21} \mathrm{~J}\right)$ 


\section{List of Figures}

1 Van der Waals constant between hydrate molecules . . . . . . . . 30

2 Van der Waals constant between an hydrate molecule and a water molecule . . . . . . . . . . . . . . . . 30

3 Residual part $R_{(\omega)}$ to calculate the ice dielectric response function 31

$4 \quad$ Ice dielectric response function (Elbaum-Seki [23] and this work) 31

5 Dielectric response functions of liquid water, ice and hydrate . . 32

6 Interaction potential between two hydrate spheres . . . . . . . . 32

$7 \quad$ Attractive force between two hydrate spheres . . . . . . . . . . 33 


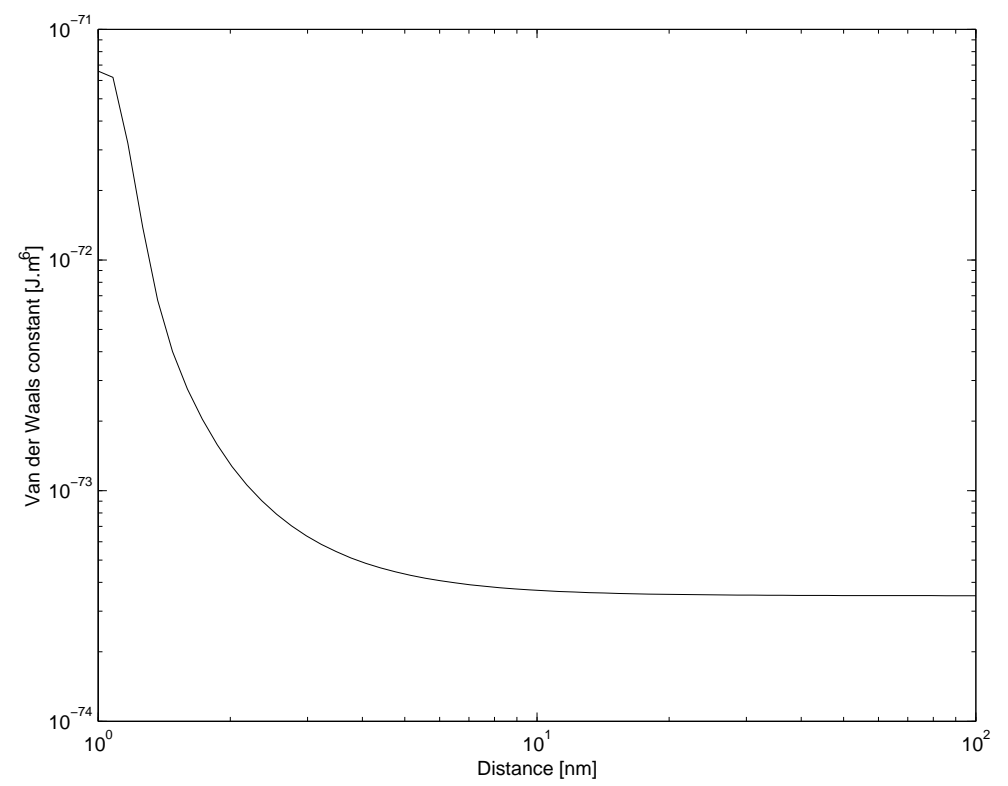

Figure 1: Van der Waals constant between hydrate molecules

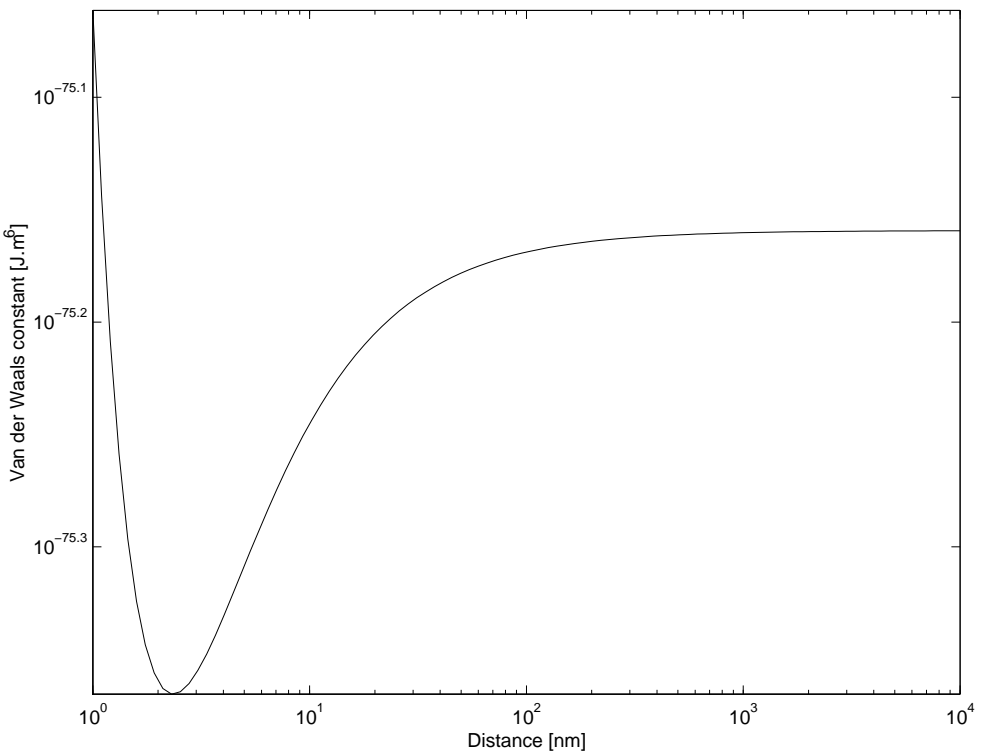

Figure 2: Van der Waals constant between an hydrate molecule and a water molecule 


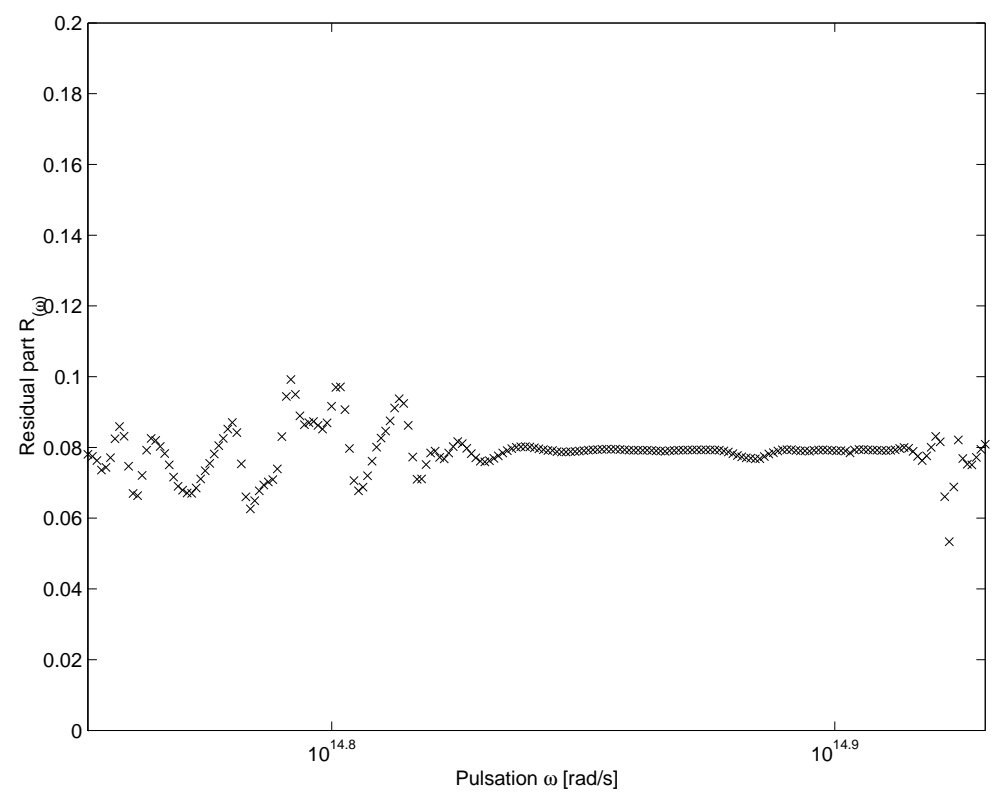

Figure 3: Residual part $R_{(\omega)}$ to calculate the ice dielectric response function

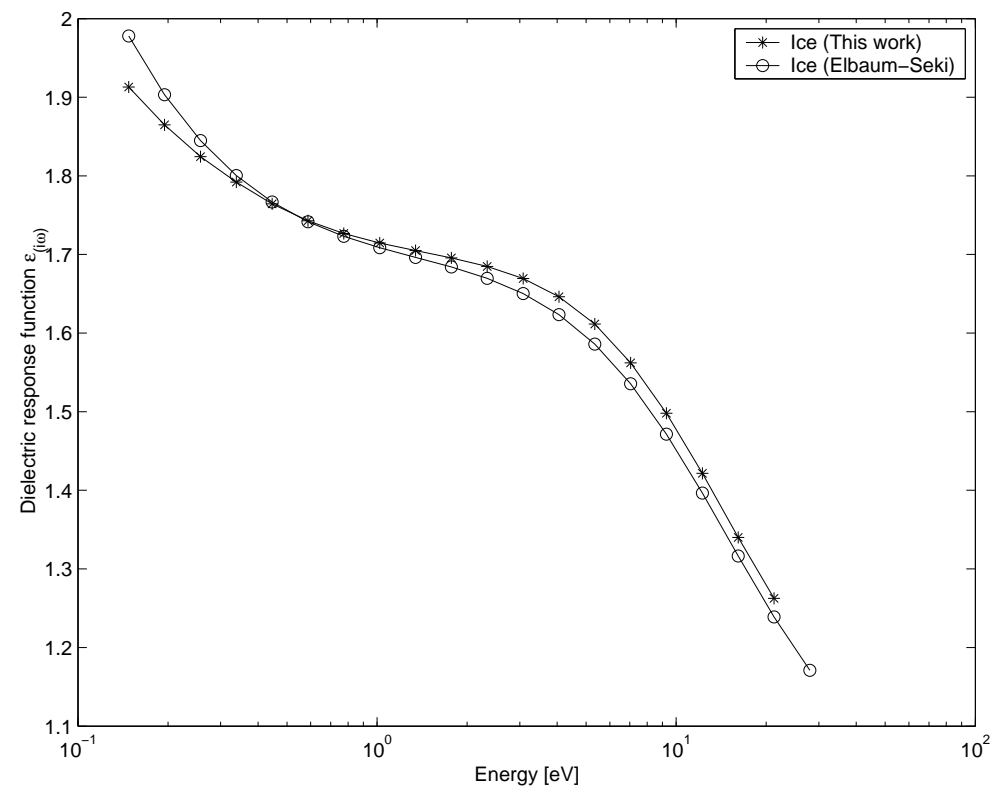

Figure 4: Ice dielectric response function (Elbaum-Seki [23] and this work) 


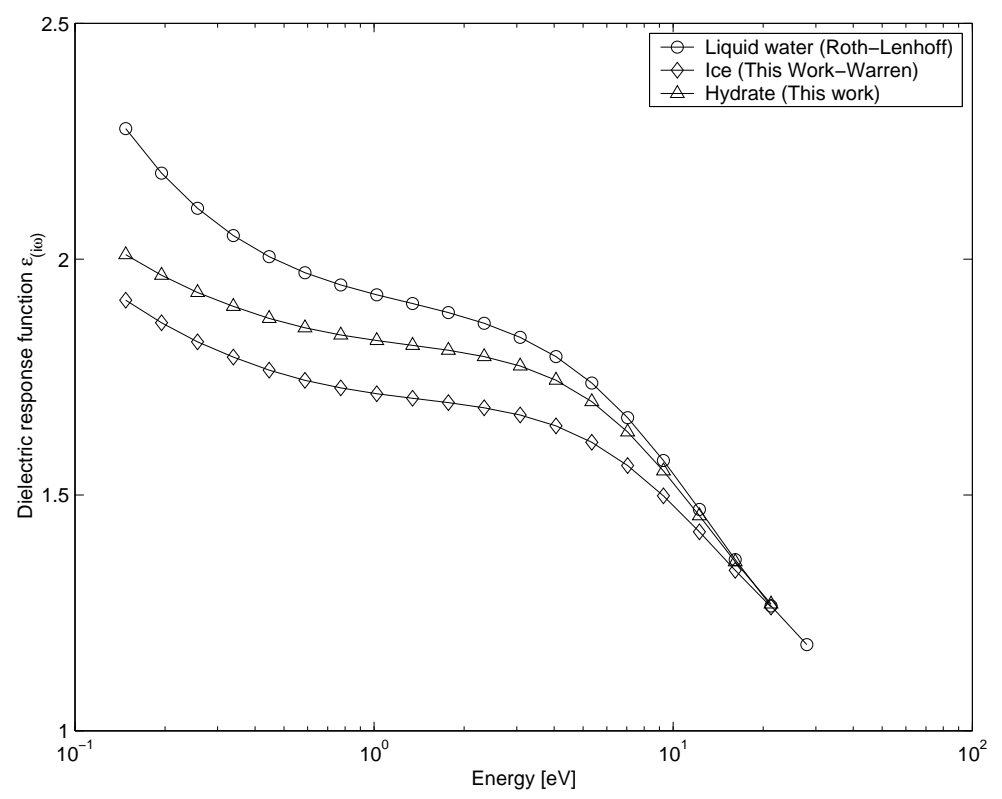

Figure 5: Dielectric response functions of liquid water, ice and hydrate

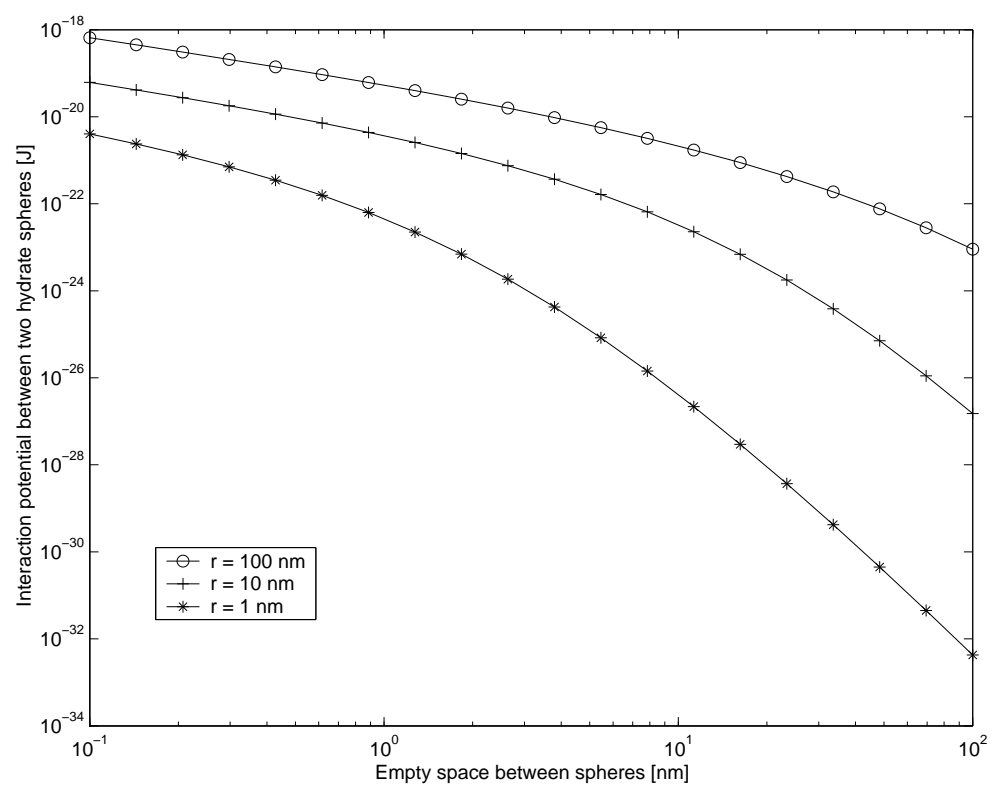

Figure 6: Interaction potential between two hydrate spheres 


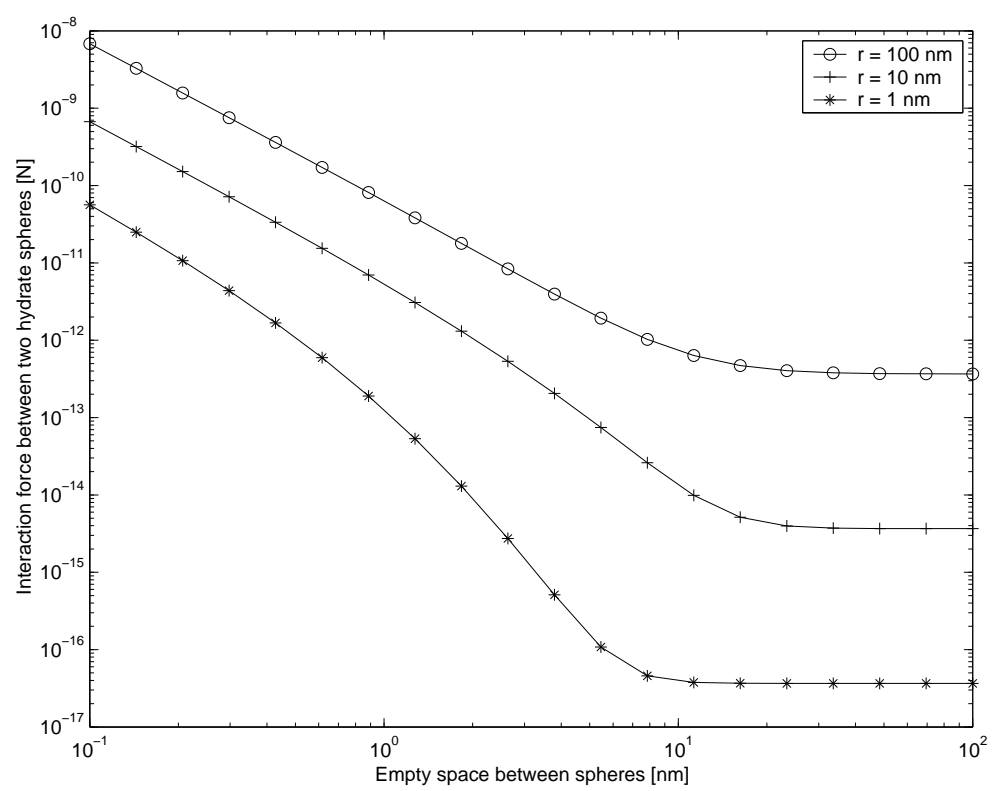

Figure 7: Attractive force between two hydrate spheres 